\title{
Optimal Offering and Operating Strategy for a Large Wind-Storage System as a Price
} Maker

Ding, Huajie; Pinson, Pierre; Hu, Zechun; Wang, Jianhui ; Song, Yonghua

Published in:

IEEE Transactions on Power Systems

Link to article, DOI:

10.1109/TPWRS.2017.2681720

Publication date:

2017

Document Version

Peer reviewed version

Link back to DTU Orbit

Citation (APA):

Ding, H., Pinson, P., Hu, Z., Wang, J., \& Song, Y. (2017). Optimal Offering and Operating Strategy for a Large Wind-Storage System as a Price Maker. IEEE Transactions on Power Systems, 32(6), 4904-4913.

https://doi.org/10.1109/TPWRS.2017.2681720

\section{General rights}

Copyright and moral rights for the publications made accessible in the public portal are retained by the authors and/or other copyright owners and it is a condition of accessing publications that users recognise and abide by the legal requirements associated with these rights.

- Users may download and print one copy of any publication from the public portal for the purpose of private study or research.

- You may not further distribute the material or use it for any profit-making activity or commercial gain

- You may freely distribute the URL identifying the publication in the public portal 


\title{
Optimal Offering and Operating Strategy for a Large Wind-Storage System as a Price Maker
}

\author{
Huajie Ding, Member, IEEE, Pierre Pinson, Senior Member, IEEE, Zechun Hu, Senior Member, IEEE \\ Jianhui Wang, Senior Member, IEEE, and Yonghua Song, Fellow, IEEE
}

\begin{abstract}
Wind farms and energy storage systems are playing increasingly more important roles in power systems, which makes their offering non-negligible in some markets. From the perspective of wind farm-energy storage systems (WF-ESS), this paper proposes an integrated strategy of day-ahead offering and real-time operation policies to maximize their overall profit. As participants with large capacity in electricity markets can influence cleared prices by strategic offering, a large scaled WFESS is assumed to be a price maker in day-ahead markets. Correspondingly, the strategy considers influence of offering quantity on cleared day-ahead prices, and adopts linear decision rules as the real time control strategy. These allow enhancing overall profits from both day-ahead and balancing markets. The integrated price-maker strategy is formulated as a stochastic programming problem, where uncertainty of wind power generation and balancing prices are taken into account in the form of scenario sets, permitting to reformulate the optimization problem as a linear program. Case studies validate the effectiveness of the proposed strategy by highlighting and quantifying benefits comparing with the price-taker strategy, and also show the profit enhancement brought to the distributed resources.
\end{abstract}

Index Terms-Electricity markets, energy storage system, linear decision rules, offering strategy, price-maker strategy, wind farm

\section{NOMENCLATURE}

\section{Indices/Sets}

$\begin{array}{ll}i \in I & \text { Index of wind farms. } \\ j \in J & \text { Index of energy storage systems } \\ t \in T & \text { Index of time intervals in a day. }\end{array}$

This research work was partly supported by the National Key Research and Development Program of China (2016YFB0900501) and the National Natural Science Foundation of China (51477082). Pierre Pinson is partly supported by the Danish Strategic Council for Strategic Research through the projects PROAIN (no. 3045-00012B/DSF) and 5s - Future Electricity Markets (no. 12-132636/DSF). J. Wang's work is supported by the U.S. Department of Energy (DOE)'s Office of Electricity Delivery and Energy Reliability under contract DE-OE0000839.

Huajie Ding was with the State Key Lab of Power Systems, Department of Electrical Engineering, Tsinghua University, Beijing, 100084, China. He is now with the State Grid Jibei Electric Power Company, Beijing, 100053, China (e-mail: hwageding@gmail.com).

Pierre Pinson is with the Department of Electrical Engineering, Technical University of Denmark, Lyngby, 2800, Denmark (e-mail ppin@elektro.dtu.dk).

Zechun $\mathrm{Hu}$ and Yonghua Song are with the State Key Lab of Power Systems, Department of Electrical Engineering, Tsinghua University, Beijing, 100084, China (e-mail: zechhu@tsinghua.edu.cn, yhsong@tsinghua.edu.cn).

Jianhui Wang is with the Department of Electrical Engineering at Southern Methodist University, Dallas, TX,75275, USA (email: jianhui@smu.edu).

\author{
$s \in S$ \\ Index of scenarios in balancing markets. \\ $b \in B$ \\ Index of blocks of discretized residual demand \\ curves.
}

\section{Parameters}

$q_{t, b}^{i n i}, q_{t, b}^{\max }$ Initial and maximal power of interval $t$, block $b$ of residual demand curve.

$\Delta t \quad$ Duration of each time interval, 1 hour.

$\eta_{\mathrm{c}}^{j}, \eta_{\mathrm{d}}^{j} \quad$ Charging and discharging efficiency of energy storage $j$.

$\bar{p}^{c, j}, \underline{p}^{c, j} \quad$ upper and lower boundary of charging power of ESS $j$.

$\bar{p}^{d, j}, \underline{p}^{d, j} \quad$ upper and lower boundary of discharging power of ESS $j$.

$\bar{E}^{j}, \underline{E}^{j} \quad$ upper and lower boundary of residual energy of ESS $j$.

$C_{i}^{w} \quad$ Capacity of wind farm $i$.

$p_{t, s}^{w f, i} \quad$ Wind power forecast of wind farm $i$ in interval $t$, scenario $s$.

$M \quad$ A large enough number

Variables

$\lambda_{t}^{b i d} \quad$ Cleared day-ahead price for interval $t$.

$\lambda_{t, s}^{b} \quad$ Balancing price for interval $t$, scenario $s$.

$p_{t}^{b i d} \quad$ Day-ahead offer of WF-ESS for interval $t$.

$q_{t, b}^{v a r} \quad$ Variable power of interval $t$, block $b$ of residual demand curve

$u_{t, b} \quad$ State variable indicating whether block $b$ of residual demand curve is chosen for interval $t$.

$p_{t, s}^{w, i} \quad$ Power output of wind farm $i$ in interval $t$, scenario $s$.

$p_{t, s}^{c, j}, p_{t, s}^{d, j} \quad$ Charging and discharging power of energy storage system $j$ in interval $t$, scenario $s$.

$p_{t, s}^{b} \quad$ Joint power output of WF-ESS in interval $t$, scenario $s$.

\section{INTRODUCTION}

W IND farms face substantial financial risks when participating competition in electricity markets owing to its lack of controllability and predictability [1]. With flexible 
charging and discharging capability, energy storage systems are deemed promising to coordinate with wind farms. The optimal offering and operating strategies of wind farm-energy storage systems (WF-ESS) have been studied a lot in recent years [2]-[5]. Most of them are based on the assumption that the capacity of WF-ESS is negligible compared to that of the whole electricity market so that its offering has little impact on the cleared prices. However, with dramatic development of WF and ESS, the scale of WF-ESS increases and consequently in some markets the WF-ESS may influence the cleared prices. Meanwhile, virtual power plant (VPP) can aggregate distributed WFs and ESS [6], [7], and take part in the competition in electricity markets as a large participant [8], [9]. Extending our former study on offering and operating strategies of WF-ESS as a price taker [1], this paper studies corresponding strategies of a large WF-ESS as a price maker in electricity markets, more specifically in energy markets. The large scaled WF-ESS can be centralized or distributed, which means the WF-ESS may consist of a large wind farm group and a large ESS such as pumped storage plant, or it may be in the form of a VPP, aggregating large quantities of distributed wind turbines and energy storages with small capacity. The essential difference between the models of a price maker and a price taker is whether influences of offers on cleared prices are considered. In this paper, the WF-ESS is assumed to be a price maker in the day-ahead market while a price taker in the balancing market [10]. Similar with in [1], the WF-ESS adopts linear decision rules as its control strategy in balancing markets, but optimizes its offering in day-ahead stages as a price maker.

Price-maker strategies are initially studied for thermal generators. Reference [11] proposed a set of regulation tools to measure the market power of oligopolistic participants in energy markets, and put forward an optimal strategy for the oligopolistic generators as a price maker within the regulatory limits. Based on this, [12] improved the model by formulating it as a mixed integer programming. Reference [13] further considered the network constraints and proposed a bi-level optimization model. The profit of thermal generators is optimized in the upper level while the lower level conducts market clearing. The obtained cleared prices are fed to the upper level through mathematical program equilibrium constraints (MPEC) and the whole model is formulated as a mixed integer programming problem. Similarly, [14] and [10] utilized MPEC to study the optimal price-maker offering strategies of wind farms in day-ahead and balancing markets respectively. Reference [15] studied the price-maker strategy of a hydrogenerator with pumped storage plant. However, the pumped storage plant in [15] is only to compensate the generation deviation from day-ahead offers of hydro-generator, which ignores its arbitrage potential. Except for the generators, massive resources of demand response can also be aggregated and then participate in the electricity market as a price maker, which is studied by [16]. Conclusively, there is few study on the offering and operating strategy of WF-ESS as a price maker as far as the authors are concerned. Furthermore, as the main topic of this paper is the price-maker strategy, the network congestion is without consideration here for the convenience of analysis, which means the cleared prices at all bus are the same. Consequently, it is not necessary to employ MPEC in this paper.

Our main contribution consists of three points. Firstly we propose a price-maker offering strategy of WF-ESS in dayahead markets, which considers the influence of offers on cleared prices. Secondly, we adopts the linear decision rules as the real-time control strategy to utilize the latest price information to make arbitrage. Meanwhile, as the key decision parameters are determined in the day-ahead stage, it is not necessary to keep high reliability for communication between the aggregator and distributed resources, which makes it suitable for distributed control. The last point is that this paper models the integrated offering-operating strategy as a mixed integer programming problem, and equivalently converts it into a linear formulation. Case studies are carried out to validate the effectiveness of proposed strategy.

The paper is organized as follows. Section II firstly introduces basic rules of day-ahead and balancing markets, and explains our price-maker offering strategy in day-ahead markets, as well as linear decision rules for balancing markets. The price-maker offering strategy and real-time operation policy based on linear decision rules are formulated as an integrated stochastic optimization problem in Section III. The objective function and constraints are further reformulated into linear ones to obtain optimal solutions. In Section IV, case studies based on historical data of wind farms and electricity markets in Denmark are carried out. Optimization results of the price-maker strategy are demonstrated and compared with those of price-taker strategies. Subsequently, taking distributed wind and storage resources for example, we analyze their profit under scenarios of separate operation, coordinated operation with price-taker strategies and coordinated operation with price-maker strategies. Finally, conclusions and perspectives for future work are gathered in Section V.

\section{Price-MAKer StRategy of WF-ESS IN ELECTRICITY MARKETS}

\section{A. Imbalance Management in Electricity Markets}

Generally deregulated electricity markets consist of dayahead and balancing/real-time market stages. Day-ahead markets allow participants to bid for their generation schedule for the whole time horizons of the following day. These are cleared 10 to 12 hours prior to the first time interval for energy delivery of the following day. Such mechanism inevitably results in deviations between scheduled and actual generation, which is particularly severe for stochastic renewable energy generators [17]. If a deviation from schedule occurs, the generator should buy or sell up/down regulation services in the balancing market [4]. For most electricity markets adopting locational marginal prices, balancing prices for up and down regulation services are the same and determined according to the overall system imbalance. More detailed descriptions and discussions on balancing markets can be found in [17]. In this paper, the models are built and illustrated based on one-price settlement in the balancing market, while the possibility of WF-ESS additionally participating ancillary service markets is without consideration. 


\section{B. Price-maker Strategy for Day-ahead Markets}

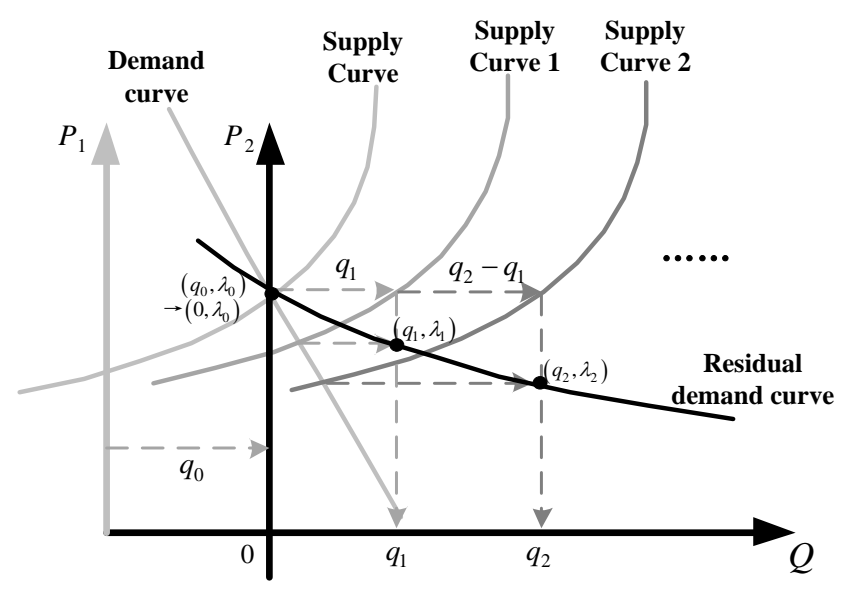

Fig. 1. Translate the supply-demand curves to the residual demand curve

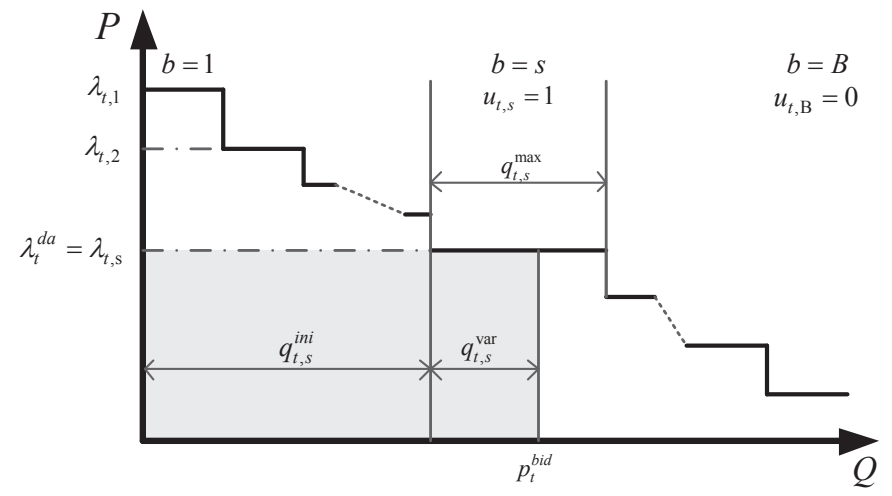

Fig. 2. An example of discretized residual demand curve

Price makers can influence the cleared quantity and price by adjusting their own offers. Residual demand curve is firstly introduced to depict this effect [12]. The supply-demand curves of day-ahead markets will be made public to all market participants after about one month by the market operator. Consequently, market participants can form the supplydemand curves for future time intervals through forecast, simulation, inverse optimization [18], etc., and translate them into residual demand curves. With all the other participants treated as whole, there is an initial cleared point by demand curve and supply curve (which does not include the offers of the target participant, namely WF-ESS). When the cleared quantity of the WF-ESS increases gradually, the supply curve shifts right, and a set of new clear points come out. As supply curves of all the participants are non-decreasing, the more the large participant bids, the lower the cleared price is, which makes the residual demand curve non-increasing. More specifically, the detailed procedure of translating supplydemand curves to residual demand curve is explained as follows. As shown in Fig. 1, the initial intersection point of supply and demand curves in coordinate system $P_{1}-Q(P$ is price and $Q$ is for quantity) is $\left(q_{0}, \lambda_{0}\right)$. Let's shift the y-axis right by $q_{0}$, then the same point is re-denoted as $\left(0, \lambda_{0}\right)$ in the coordinate system $P_{2}-Q$. It means if the offering quantity of WF-ESS were 0 , then the cleared price would be $\lambda_{0}$. If the supply curve were shifted right by $q_{1}$, then the new supply curve and demand curve would generate a new intersection point whose $\mathrm{y}$-value is $\lambda_{1}$. A new point $\left(q_{1}, \lambda_{1}\right)$ means if the cleared quantity of WF-ESS were $q_{1}$, then the cleared price would be $\lambda_{1}$. If the supply curve were shifted right by $q_{2}$, $q_{3} \cdots$, and so forth, then there would be a series of points $\left(q_{k}, \lambda_{k}\right)$, and these points form the residual demand curve.

The residual demand curve is usually in discretized form for convenience of formulation. As shown in Fig. 2, the discretized residual demand curve has $B$ blocks. The offering quantity and price can be modeled as linear formulation by introducing binary variables.

For any time period $t \in T$, there are:

$$
\begin{gathered}
\sum_{b \in B} u_{t, b}=1 \quad u_{t, b} \in\{0,1\} \\
0 \leq q_{t, b}^{v a r} \leq u_{t, b} q_{t, b}^{\max } \\
p_{t}^{b i d}=\sum_{b \in B}\left(u_{t, b} q_{t, b}^{i n i}+q_{t, b}^{v a r}\right) \\
\lambda_{t}^{d a}=\sum_{b \in B} u_{t, b} \lambda_{t, b}^{d a}
\end{gathered}
$$

where $q_{t, b}^{i n i}, q_{t, b}^{v a r}$ and $q_{t, b}^{\max }$ indicate the initial, variable and maximal power of interval $t$, block $b$, while $\lambda_{t, b}^{d a}$ is the corresponding price in day-ahead market. The decision variable $u_{t, b}$ indicates whether the participant will choose block $b$ as its offering price and quantity.

Equation (1a) limits the range of offering price and quantity within the residual demand curve, and the variable power of every interval $t$ block $b$ should be within specific ranges, as required by (1b). Equation (1c) determines the day-ahead offering quantity through $u_{t, b}$ and $q_{t, b}^{v a r}$. As shown in Fig. 2, when block $s$ is chosen, then $u_{t, s}=1$. The corresponding offering quantity $p_{t}^{b i d}$ should be the sum of the initial power $q_{t, s}^{i n i}$ and the variable power $q_{t, s}^{v a r}$ of block $s$. For any interval $t$, as only one block has its state variable $u_{t, b}=1$, then the sum of $\lambda_{t, b}^{d a} u_{t, b}$ of all blocks would be the offering price, as (1d) indicates.

\section{Linear Decision Rules for Balancing Markets}

The strategy of linear decision rules for balancing markets has been introduced in detail previously [1], but in order to be self-consistent, it is briefly explained here. The whole time horizon can be discretized into $T$ time intervals, and the state vector $\boldsymbol{x}=\left[x_{1} \ldots x_{T}\right]^{\top} \in \mathbb{R}^{T}$ stands for the residual energy of ESS at the end of each time interval. The state variables are temporally coupled as

$$
x_{t}=x_{t-1}+\boldsymbol{B} \boldsymbol{u}_{t}, \quad t=1,2, \ldots T
$$

where $\boldsymbol{B}=\left[\eta_{c},-1 / \eta_{d}\right]$ and $x_{0}$ is the initial residual energy. $\boldsymbol{u}_{t}=\left[p_{t}^{c}, p_{t}^{d}\right]^{\top}$ consists of the $t$ th elements of charging power vector $\boldsymbol{p}^{c} \in \mathbb{R}^{\mathrm{T}}$ and discharging power vector $\boldsymbol{p}^{d} \in \mathbb{R}^{\mathrm{T}} . \eta_{c}$ and $\eta_{d}$ are charging and discharging efficiencies of the ESS. In this paper, linear decision rules determine the charging and discharging power vectors of ESS by affine functions of dayahead and balancing price forecast error, i.e., 


$$
\left[\begin{array}{l}
\boldsymbol{p}^{c} \\
\boldsymbol{p}^{d}
\end{array}\right]=\left[\begin{array}{l}
\widehat{\boldsymbol{p}}^{c} \\
\widehat{\boldsymbol{p}}^{d}
\end{array}\right]+\left[\begin{array}{ll}
\boldsymbol{D}_{d a}^{c} & \boldsymbol{D}_{r t}^{c} \\
\boldsymbol{D}_{d a}^{d} & \boldsymbol{D}_{r t}^{d}
\end{array}\right]\left[\begin{array}{c}
\boldsymbol{\Delta} \boldsymbol{\pi}^{d a} \\
\boldsymbol{\Delta} \boldsymbol{\pi}^{r t}
\end{array}\right]
$$

and can be denoted as $\boldsymbol{p}=\widehat{\boldsymbol{p}}+\boldsymbol{D} \boldsymbol{\delta}$, where $\widehat{\boldsymbol{p}}$ and $\boldsymbol{D}$ are decision variables of linear decision rules.$\widehat{p}$ is the nominal power vector and $\delta$ is the vector consisting of forecast error for day-ahead prices $\Delta \boldsymbol{\pi}^{d a}$ and balancing prices $\Delta \boldsymbol{\pi}^{r t}$. The affine matrix $\boldsymbol{D}$ consists of 4 sub-matrices, which link charging and discharging power adjustments of ESS with forecast error of day-ahead and balancing prices. In the day-ahead optimization, as the forecast of wind power and prices are formulated by scenarios, the forecast error in (3) is the difference between the value of each scenario and the mean value.

\section{Formulation OF the InTEgRATED STRATEGy}

Wind farms and energy storage systems coordinate to maximize their overall revenue. Without considering their preferences on risks, the optimization model for the pricemaker strategy can be formulated as:

$$
\begin{array}{cl}
\max _{\boldsymbol{\theta}} & \mathbb{E}(\widetilde{S}(\boldsymbol{\theta}, \boldsymbol{\delta})) \\
\text { s.t. } & g(\boldsymbol{\theta}, \boldsymbol{\delta}) \leq 0
\end{array}
$$

where $\boldsymbol{\theta}=\left\{\boldsymbol{\lambda}^{d a}, \boldsymbol{p}^{b i d}, \boldsymbol{D}, \widehat{\boldsymbol{p}}\right\}$ are decision variables, $\boldsymbol{\delta}$ is the vector consisting of forecast errors of wind power, day-ahead and balancing prices. $\boldsymbol{p}^{\text {bid }}$ is the vector of day-ahead offers $\left\{p_{t}^{b i d}\right\}$, and $\boldsymbol{\lambda}^{d a}$ is the vector of day-ahead cleared prices $\left\{\lambda_{t}^{d a}\right\}$. All the offers whose prices are under the marginal price can be cleared out. The objective function (4a) maximizes the expected profit of WF-ESS, while the constraints (4b) limit the power output of wind farms and energy storage systems, as well as the residual energy of energy storage systems.

\section{A. Details of the Objective Function}

The overall profit of WF-ESS includes that from day-ahead markets and from balancing markets. As the wind power output is uncertain, the profit in balancing markets is modeled in form of expected value. More specifically, if the wind power uncertainty is depicted as scenarios, then the expected profit is the weighted sum of profit of all scenarios. The expected overall profit of WF-ESS can be formulated as follows.

$$
\begin{aligned}
\mathbb{E}(\widetilde{S}(\boldsymbol{\theta}, \boldsymbol{\delta})) & =\sum_{t \in T}\left\{R_{t}^{d a}+\sum_{s \in S} \rho_{s} R_{t, s}^{b}\right\} \\
R_{t}^{d a} & =\lambda_{t}^{d a}\left(p_{t}^{b i d}\right) \cdot p_{t}^{b i d} \\
-\sum_{j \in J} \bar{p}_{j}^{c} & \leq p_{t}^{b i d} \leq \sum_{i \in I} C_{i}^{w}+\sum_{j \in J} \bar{p}_{j}^{d} \\
p_{t, s}^{b} & =\sum_{i \in I} p_{t, s}^{w, i}+\sum_{j \in J} p_{t, s}^{d, j}-\sum_{j \in J} p_{t, s}^{c, j} \\
R_{t, s}^{b} & =\lambda_{t, s}^{b} \cdot\left(p_{t, s}^{b}-p_{t}^{b i d}\right)
\end{aligned}
$$

Day-ahead prices $\lambda_{t}^{d a}$ are determined by the day-ahead offering quantity $p_{t}^{b i d}$, obeying the residual demand curve. The product of day-ahead price and quantity (the shadow part in Fig. 2) is the profit in day-ahead markets $R_{t}^{d a}$. The upper bound of day-ahead offers is the capacity of WF-ESS, while the lower bound is the charging capacity of all ESS as (5c). In balancing markets, the uncertain wind power is modeled though scenarios $s \in S$, whose weight is $\rho_{s}$. The joint power output of WF-ESS is as (5d), where $i \in I$ is the index of every wind farms, $j \in J$ is the index of every energy storage systems. $p_{t, s}^{w, i}, p_{t, s}^{c, j}$ and $p_{t, s}^{d, j}$ are the power of wind farm $i$, and charging/discharging power of energy storage system $j$ in interval $t$, scenario $s$. The joint power output is the sum of the power from all the wind farms and energy storage systems. $\lambda_{t, s}^{b}$ is the balancing price of interval $t$, scenario $s$. The difference between the joint power output of WFESS in the balancing market and the day-ahead market is the offered up/down-regulation services. Multiplying the up/downregulation capacity by the balancing prices gets the profit in balancing markets. Consequently, $R_{t, s}^{b}$ is the profit of WFESS from balancing markets in interval $t$, scenario $s$. As the WF-ESS is a price taker in balancing markets, the balancing prices are independent from the up/down-regulation capacity, as formulated by (5e).

It is worth noting that the day-ahead price (1d) and quantity (1c) both have decision variables $u_{t, b}$ and $q_{t, b}^{v a r}$. Consequently, the day-ahead profit (5b) is a quadratic formulation. According to the property of binary variables, they can be equivalently transformed to a mixed integer linear formulation.

$$
R_{t}^{d a}=\sum_{b \in B} \lambda_{t, b}^{d a}\left(u_{t, b} q_{t, b}^{i n i}+q_{t, b}^{v a r}\right)
$$

\section{B. Details of the Constraints}

In real-time operation, wind farms can benefit from maximal power generating as long as the balancing price is higher than their operation cost, which is slightly higher than 0 . However, the charging and discharging power of ESS is temporally coupled due to limited residual energy, the ESS needs to optimize its charging and discharging power of each time interval. Real-time control strategy based on linear decision rules can fully utilize the historical and current market information, as well as the forecast results for the future intervals, to obtain the optimal control decision. In this paper, key parameters of linear decision rules are determined in day-ahead optimization and used for real-time operation. The constraints for charging/discharging power and related residual energy of ESS can be formulated as:

$$
\begin{aligned}
& p_{t, s}^{c, j}=u_{t}^{c, j} \sum_{k \in T} D_{t, k}^{c, j} \lambda_{k, s}^{b}+\hat{p}_{t}^{c, j} \quad \forall j \in J \\
& p_{t, s}^{d, j}=u_{t}^{d, j} \sum_{k \in T} D_{t, k}^{d, j} \lambda_{k, s}^{b}+\hat{p}_{t}^{d, j} \quad \forall j \in J \\
& E_{t, s}^{j}=E_{t-1, s}^{j}+p_{t, s}^{c, j} \Delta t \eta_{c}^{j}-p_{t, s}^{d, j} \Delta t \eta_{d}^{j} \quad \forall j \in J
\end{aligned}
$$

where $u_{t}^{c, j}$ and $u_{t}^{d, j}$ are binary decision variables indicating charging and discharging state of ESS $j$ in interval $t . \hat{p}_{t}^{c, j}$ and $\hat{p}_{t}^{d, j}$ are the $t$ th element of $\widehat{\boldsymbol{p}}^{c}$ and $\widehat{\boldsymbol{p}}^{d}$ for ESS $j . \mathbf{D}^{c, j}, \mathbf{D}^{d, j}$ are affine matrices of linear decision rules for ESS $j . D_{t, k}^{c, j}$ in (7a) is the value of $\mathbf{D}^{c, j}$ at $(\mathrm{t}, \mathrm{k}) . \lambda_{k, s}^{b}$ is the balancing price of interval $k$, scenario $s$, which is similar with (7b). Equation (7c) shows the temporal coupling of ESS residual energy in each time interval. 
Real-time operation of wind farms and energy storage systems should satisfy related constraints, including:

$$
\begin{gathered}
0 \leq p_{t, s}^{w, i} \leq p_{t, s}^{w f, i} \quad \forall i \in I \\
u_{t}^{c, j}+u_{t}^{d, j} \leq 1 \quad \forall j \in J \\
\underline{p}^{c, j} \leq \sum_{k \in T} D_{t, k}^{c, j} \lambda_{k, s}^{b}+\hat{p}_{t}^{c, j} \leq \bar{p}^{c, j} \quad \forall j \in J \\
\underline{p}^{d, j} \leq \sum_{k \in T} D_{t, k}^{d, j} \lambda_{k, s}^{b}+\hat{p}_{t}^{d, j} \leq \bar{p}^{d, j} \quad \forall j \in J \\
\underline{E}^{j} \leq E_{t, s}^{j} \leq \bar{E}^{j} \quad \forall j \in J
\end{gathered}
$$

where $\bar{p}^{c, j}$ and $p^{c, j}$ are upper and lower charging boundaries of ESS $j, \bar{p}^{d, j}$ and $p^{d, j}$ are discharging boundaries. $\bar{E}^{j}$ and $E^{j}$ are upper and lower boundaries of residual energy of ESS. Equation (8a) limits the power output of wind farm $i$ under the forecast value $p_{t, s}^{w f, i}$. Equation (8b) forbids simultaneous charging and discharging of ESS. Charging power $p_{t, s}^{c, j}$, discharging power $p_{t, s}^{d, j}$, as well as the the residual energy $E_{t, s}^{j}$ of ESS $j$ should satisfy the operation constraints as shown in (8c)-(8e). Except for the constraints of day-ahead optimization, at interval $t$ in balancing periods, the charging upper bound is set as $\min \left\{\bar{p}_{c}, \frac{E_{\max }-E_{t}}{\eta_{c} \Delta t}\right\}$, and the discharging upper bound is set as $\min \left\{\bar{p}_{d}, \frac{E_{t}-E_{\min }}{\Delta t} \eta_{d}\right\}$, guaranteeing the residual energy in allowable ranges at next time interval.

Equation (7a) and (7b) are both quadratic, and can be transformed into linear ones through reformulation. Equation (7a) is reformulated as

$$
\left\{\begin{array}{l}
0 \leq p_{t, s}^{c, j} \leq \sum_{k \in T} D_{t, k}^{c, j} \lambda_{k, s}^{b}+\hat{p}_{t}^{c, j} \\
\sum_{k \in T} D_{t, k}^{c, j} \lambda_{k, s}^{b}+\hat{p}_{t}^{c, j}-\left(1-u_{t}^{c, j}\right) M \leq p_{t, s}^{c, j} \leq u_{t}^{c, j} M
\end{array}\right.
$$

Equation (7b) is reformulated as:

$$
\left\{\begin{array}{l}
0 \leq p_{t, s}^{d, j} \leq \sum_{k \in T} D_{t, k}^{d, j} \lambda_{k, s}^{b}+\hat{p}_{t}^{d, j} \\
\sum_{k \in T} D_{t, k}^{d, j} \lambda_{k, s}^{b}+\hat{p}_{t}^{d, j}-\left(1-u_{t}^{d, j}\right) M \leq p_{t, s}^{d, j} \leq u_{t}^{d, j} M
\end{array}\right.
$$

where $M$ is a large-enough constant number. Take charging power for example, when $u_{t}^{c, j}=0$, the second row of (9) will be $p_{t, s}^{c, j} \leq 0$, coordinating with the first row $0 \leq p_{t, s}^{c, j}$ can get $p_{t, s}^{c, j}=0$. When $u_{t}^{c, j}=1$, the second row of (9) is $\sum_{k \in T} D_{t, k}^{c, j} \lambda_{k, s}^{b}+\hat{p}_{t}^{c, j} \leq p_{t, s}^{c, j}$, coordinating with the first row can get $p_{t, s}^{c, j}=\sum_{k \in T} D_{t, k}^{c, j} \lambda_{k, s}^{b}+\hat{p}_{t}^{c, j}$. The reformulation of discharging power is similar.

\section{Application Results}

\section{A. Case Design}

In this section, a WF-ESS consisting of a wind farm group with capacity of $1.0 \mathrm{GW}$ and a large energy storage with capacity of 500MW (such as a pumped storage plant) is taken for example. Realistic wind power data are from wind farms in Denmark [19], [20]. Per-unit data of wind power forecast scenarios are provided by [21] and translated into actual data by multiplying $C_{w}=1000 \mathrm{MW}$. More scenarios of wind power which obey the same distribution can be generated by the methodology proposed in [4] for simulation. Key parameters of WF-ESS is listed in Tab.I.
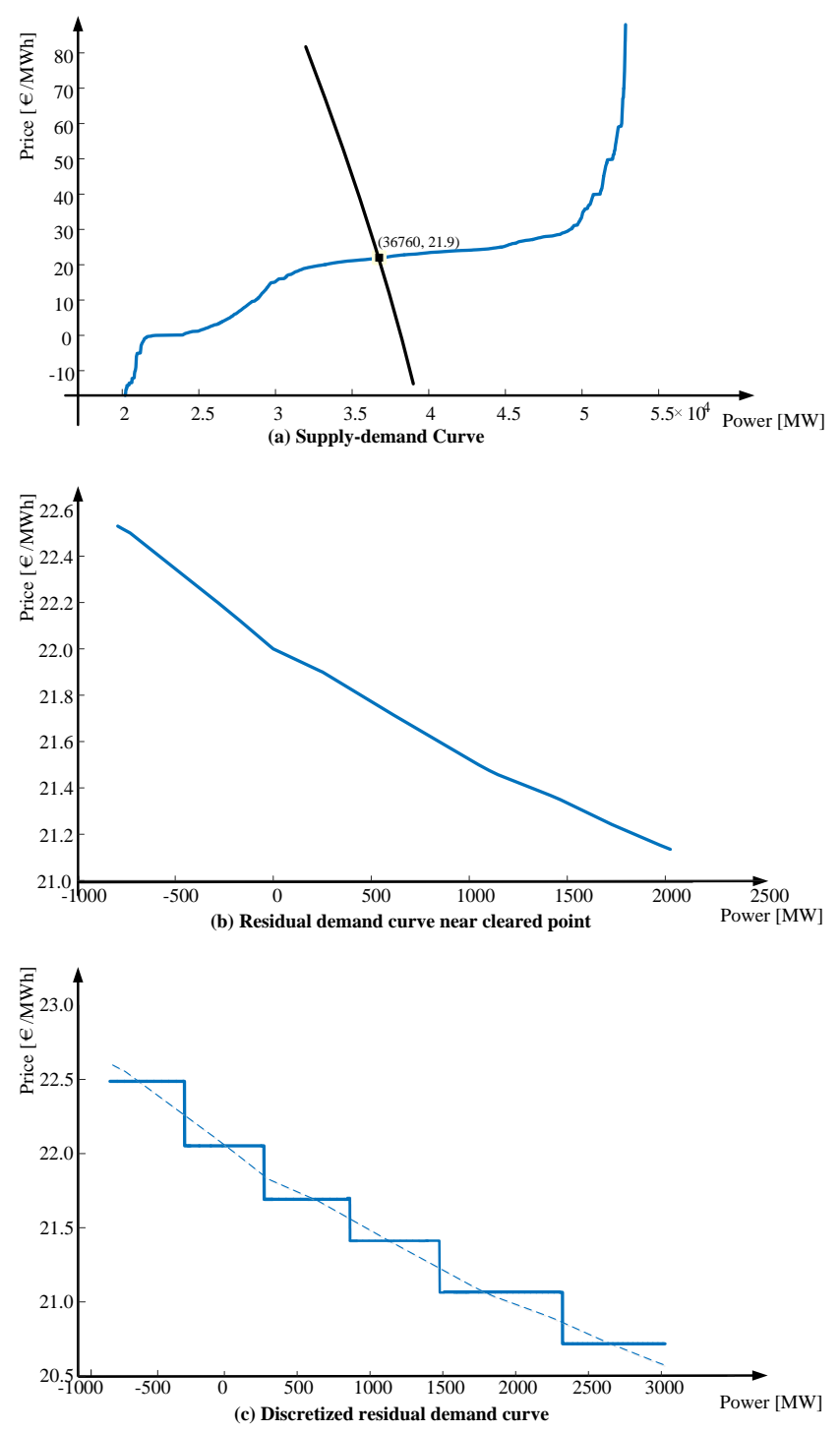

Fig. 3. From supply-demand curve to discretized residual demand curve

TABLE I

Key PARAmeters OF THE WF-ESS System

\begin{tabular}{cccc}
\hline$\eta_{c}$ & $\eta_{d}$ & $\underline{E}[\mathrm{MWh}]$ & $\bar{E}[\mathrm{MWh}]$ \\
0.9 & 0.9 & 500 & 2250 \\
\hline$E_{0}$ & $\bar{p}^{c}[\mathrm{MW}]$ & $\bar{p}^{d}[\mathrm{MW}]$ & $C_{w}[\mathrm{MW}]$ \\
1400 & 500 & 500 & 1000 \\
\hline
\end{tabular}

The residual demand curves are obtained through the realistic supply-demand curves of Nord Pool on November 11th, 2015 [22]. Take the time interval 23:00-24:00 for example, the supply-demand curves are shown in Fig.3(a). The cleared quantity is $36.76 \mathrm{GW}$ while the cleared price is $21.9 € / \mathrm{MWh}$. The corresponding residual demand curve at neighbor of cleared point is as Fig.3(b). For convenience of formulation, 
the original residual demand curve can be discretized into 6 blocks (the number of blocks is set to be 6 here for illustration, it can be any positive integer actually), and the discretized residual demand curve is as Fig.3(c). Day-ahead supplydemand curve forecasts are assumed to be accurate, so the uncertainty of residual demand curve is without consideration in this paper.

Balancing prices are also form Nord Pool markets. But as the Nord Pool is of two-price balancing market, up/downregulation prices are different and one or the other of them is equal to the day-ahead price at any specific time interval. So we take the different one as the balancing price in the oneprice balancing market. The uncertainty of balancing prices is simulated by adding the realistic data by stochastic forecast error as follows:

$$
\widetilde{\pi}_{t}^{r t}=\bar{\pi}_{t}^{r t}\left(1+\sigma_{r t} \widetilde{\varepsilon}\right)
$$

where $\bar{\pi}_{t}^{r t}$ is the historical balancing price of interval $t$. $\widetilde{\varepsilon}$ is stochastic error which obeys norm distribution $\widetilde{\varepsilon} \sim N(0,1)$, and $\sigma_{r t}$ is standard deviation of balancing prices forecasts. The uncertainty of balancing prices can be depicted by setting $\sigma_{r t}$. In this paper, $\sigma_{r t}$ is set as 0.1 . As the original day-ahead prices and balancing prices are realistic data from Nord Pool, and the cleared day-ahead prices as well as the simulated balancing prices are fluctuating slightly around the original data, the correlation between day-ahead and balancing prices can be remained to a large extent.

In case studies below, uncertainty of wind power and balancing prices are depicted by scenarios. Scenarios of balancing prices for optimization and simulation are generated by Eq.(11) separately and independently. Wind power scenarios for optimization is from [19], [20], while those for simulation are generated obeying the same distribution, as done in [4].

\section{B. Optimization Results}

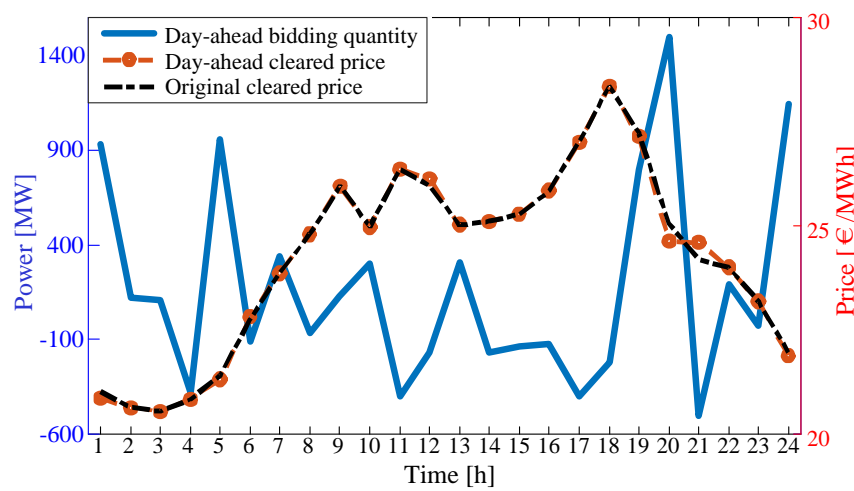

Fig. 4. The optimal offering of WF-ESS and cleared prices in day-ahead markets

The presented algorithm and model have been implemented in Matlab 2016, and solved by the commercial solver Cplex 12.5. The problem is solved in Windows 10 in about 6 seconds in Intel(R) Core(TM) i7-6600u CPU @ 2.81GHz, with $20 \mathrm{~GB}$ RAM memory.
The optimal offering and operation strategy of WF-ESS can be acquired through solving the optimization programming problem, and the results are shown in Fig.4 and Fig.5. In Fig.4, the black curve shows the original cleared prices in the day-ahead market without WF-ESS, while the brown dotted curve represents the cleared day-ahead prices with WF-ESS participating as a price maker. By comparison it is clear that in interval 1:00, 5:00 and 20:00, the offering of WF-ESS exceeds 900MW, which results in the decrease of cleared prices; in interval 11:00, 21:00, the consumption bid (negative offering) is about $500 \mathrm{MW}$, which increases the cleared prices. The influence of offering quantity on cleared prices shows the price-maker effect of large scaled WF-ESS. Fig.5 shows the power output of WF-ESS at balancing stage in one specific scenario. As a price taker in balancing markets, WF-ESS offers up-regulation capacity when balancing prices are high and offers down-regulation capacity when balancing prices are low.

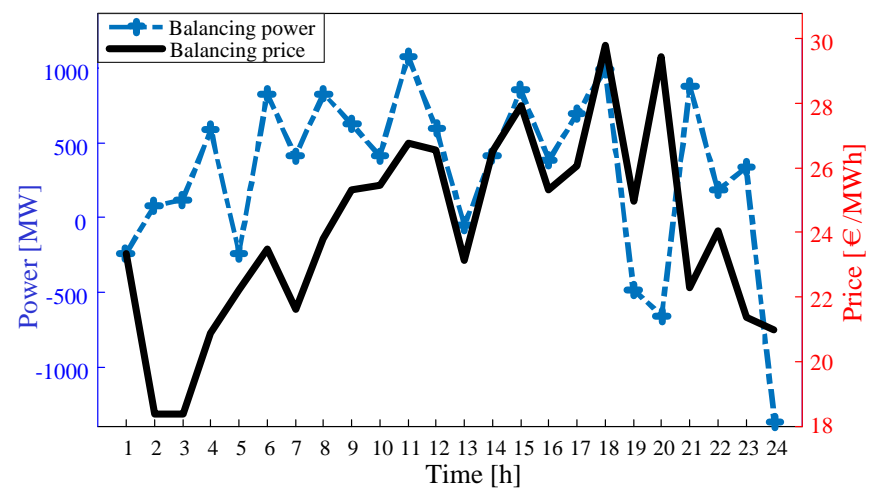

Fig. 5. The power output adjustment of WF-ESS and prices in balancing markets

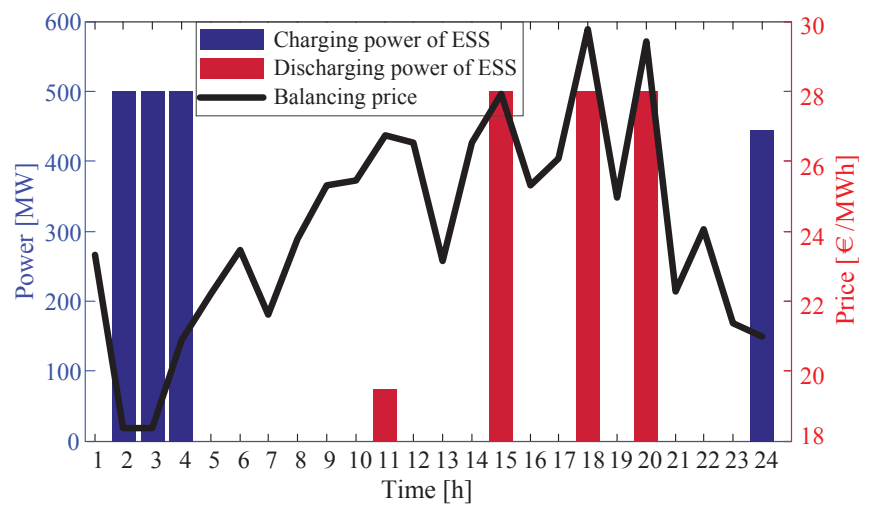

Fig. 6. Charging and discharging states of ESS in balancing markets

Fig.6 shows the charging and discharging power of ESS in balancing stages. It is obvious from the figure that ESS shifts to discharging state when balancing prices go up and shifts to charging state when balancing prices go down. As the ESS adopts linear decision rules as its operation strategy, it adjusts charging and discharging power flexibly regarding to the fluctuating balancing prices. For example, though the balancing price of the 11th time interval is higher than those of all former intervals, the ESS discharges at below 100MW to 
reserve energy for higher prices in future periods (for example 15:00, 18:00 and 20:00). It is similar for charging cases.

The optimization results show that the day-ahead profit is $91.55 \mathrm{k} €$, while the expected profit in the balancing market is $190.43 \mathrm{k} €$, and thus the overall profit of WF-ESS for a single day is $281.98 \mathrm{k} €$. It means when the WF-ESS participates in the day-ahead market as a price maker, the main profit comes from the balancing market. This result comes from the fact that the capacity of WF-ESS is large enough to influence the market clearance, which makes the WF-ESS reduce its day-ahead offering in order to avoid large price decease. More detailed explanation is given in next subsection, where comparison between price-taker and price-maker strategies is carried out.

\section{Comparison of Price-maker and Price-taker Strategies}

We firstly set up a model for a price-taker strategy of WFESS, and its solution is compared with that of the proposed price-maker strategy. As the maximal charging and discharging power of ESS is $500 \mathrm{MW}$, and the maximal capacity of WF is $1000 \mathrm{MW}$, the offering range of WF-ESS is set as $[-500$, 1500]. Real-time control strategy for price-taker strategy is still linear decision rules, the same with that of the pricemaker strategy. For convenience of statement, the price-maker strategy is abbreviated as PM strategy, while the price-taker strategy is as PT strategy. The formulation of PT strategy is as follows.

$$
\begin{array}{ll}
\max _{\boldsymbol{\theta}} & \sum_{t \in T}\left\{R_{t}^{d a}+\sum_{s \in S} \rho_{s} R_{t, s}^{b}\right\} \\
\text { s.t. } & R_{t}^{d a}=\lambda_{t}^{d a} p_{t}^{b i d} \\
& -500 \leq p_{t}^{b i d} \leq 1500 \\
& p_{t, s}^{b}=p_{t, s}^{w}+p_{t, s}^{d}-p_{t, s}^{c} \\
& R_{t, s}^{b}=\lambda_{t, s}^{b} \cdot\left(p_{t, s}^{b}-p_{t}^{b i d}\right) \\
& (7 \mathrm{a})-(10)
\end{array}
$$

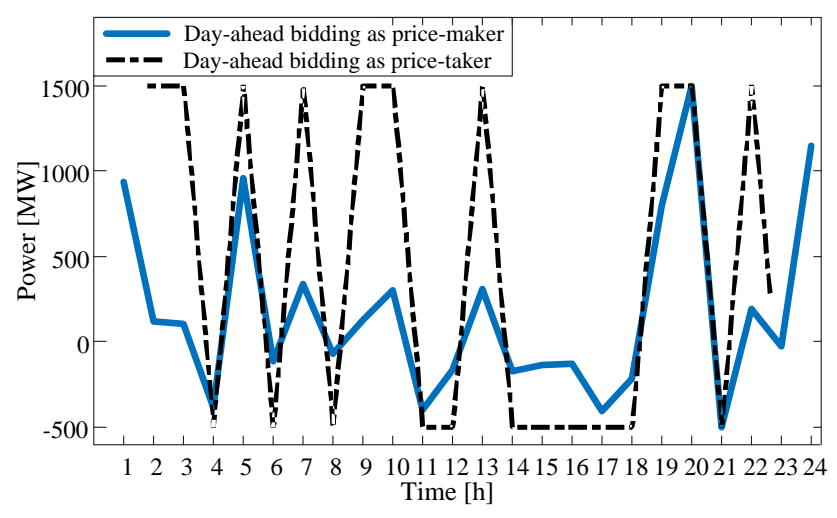

Fig. 7. Day-ahead offers of PT and PM strategies

Offering of the PT strategy is shown as the black curve in Fig.7. It is more extreme than that of PT strategy (shown as blue curve), and always touches the upper bound (1500MW) and lower bound (-500MW) of offering. From (13a)-(13c) we can see that the day-ahead offers of PT strategy depends on

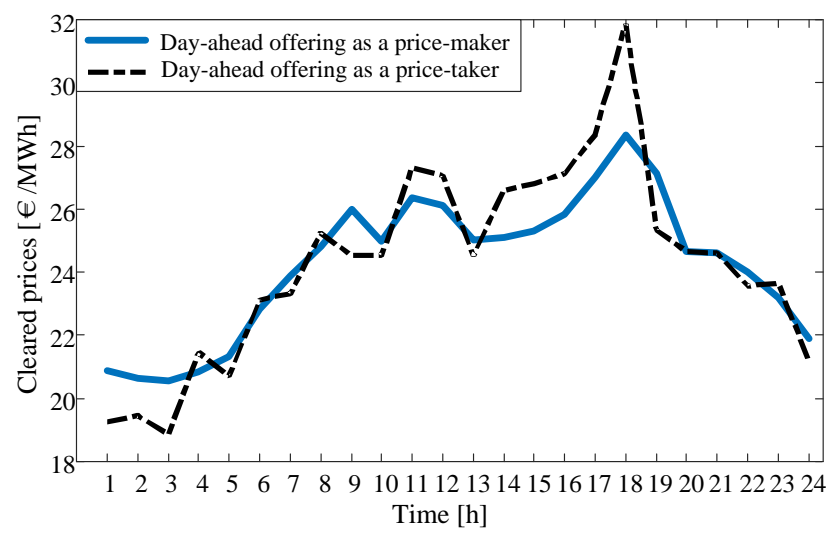

Fig. 8. Cleared prices of the day-ahead market with WF-ESS participating as a price taker or a price maker

the deviation between day-ahead price and expected balancing prices. For each time interval, if expected balancing price is higher than day-ahead price, the WF-ESS offers the lower bound and vice versa.

$$
\begin{aligned}
& R_{t}^{d a}+\sum_{s \in S} \rho_{s} R_{t, s}^{b} \quad \forall t \in T \\
= & \lambda_{t}^{d a} p_{t}^{b i d}+\sum_{s \in S} \rho_{s} \lambda_{t, s}^{b}\left(p_{t, s}^{b}-p_{t}^{b i d}\right) \\
= & \left(\lambda_{t}^{d a}-\mathbb{E}\left(\lambda_{t}^{b}\right)\right) p_{t}^{b i d}+\sum_{s \in S} \rho_{s} \lambda_{t, s}^{b} p_{t, s}^{b}
\end{aligned}
$$

The objective function value of PT strategy is $284.29 \mathrm{k} €$, which is even higher than that of PM strategy $(278.24 \mathrm{k} €)$. However, with the influence of offering quantity on cleared price considered (substituting the optimized offers into Fig. $3(\mathrm{c})$ ), the cleared price with PT strategy is shown as the black curve in Fig.8. In comparison, the cleared price with PM strategy (blue curve) is more smooth. Simultaneously referring to Fig.7, one can see that when WF-ESS bids negative capacity, the cleared price of PT strategy is higher than that of PM strategy; and when WF-ESS offers positive capacity, the cleared price of PT strategy is lower than that of PM strategy. Consequently, the actual expected day-ahead profit of PM strategy is lower than that in the objective function. The calculation and simulation results of PT and PM strategies are listed in Tab.II.

TABLE II

COMPARISON OF RESULTS FROM PT AND PM STRATEGIES $(\mathrm{k} €$ )

\begin{tabular}{cccc}
\hline item & Day-ahead Profit & Balancing Profit & Overall Profit \\
\hline PM Obj & 91.55 & 186.69 & 278.24 \\
PM Simulation & 91.55 & 190.43 & 281.98 \\
PT Obj & 272.76 & 11.52 & 284.29 \\
PT Simulation & 248.14 & -18.83 & 229.31 \\
\hline
\end{tabular}

This table lists objective function value and simulation results of PM strategy (the 2nd and 3rd row) and PT strategy (the 4th and 5th row). By comparing the results in Row 4 and Row 5, it can be seen that for day-ahead profit of PT strategy, the simulated value is $9.0 \%$ lower than that in 
objective function, and balancing profit decreases by $272 \%$. However, as the PM strategy considers the effect of offering quantity on cleared price, the day-ahead profit is the same for simulation and objective function results, and the simulated balancing profit increases by $2.0 \%$ compared to the objective function (comparing Row 2 and Row 3). The results in Row 3 and Row 5 show the PM strategy can bring 23\% higher profit than PT strategy.

It is interesting to see that over $60 \%$ profit of WF-ESS comes from the balancing market when price-maker strategy is employed, which is uncommon for conventional generators. However, it is reasonable for WF-ESS. As ESS is flexible in charging and discharging, it can make arbitrage by absorbing low-cost wind energy and selling it in balancing markets. Furthermore, WF-ESS may reserve some generation capacity in the day-ahead market and then release it when balancing price is higher, or conversely reserve some consumption capacity in the day-ahead market and fulfill it when balancing price is lower.

\section{Profit Analyses}

Except for in form of a union of a single large-scaled wind farm (group) with a large pumped storage plant, the WF-ESS can also consist of a large quantity of distributed wind turbines and battery storages. These distributed resources are aggregated and participate in the market as a VPP. VPP can help increase the competition of distributed resources in markets and hence enhance their profit. In this subsection, we assume there are 10 small wind farms and 10 distributed energy storages, whose capacity is only $10 \%$ of that in Subsection IV-A. When the capacity of WF and ESS is small, they can only compete as a price taker if participating the market separately. Here we compare the profit of WF-ESS in three cases:

Without Aggregation. The distributed WFs and ESS participate in the market separately as price takers. Then their day-ahead offers are taken into account to determine the dayahead price. Wind power and balancing prices are simulated to calculate the profit of each resource based on their offering and operation strategy. Furtherly, we also consider the case where a wind farm and a storage coordinates.

Aggregation with PT strategy. Aggregating the 10 small wind farms into a united one, and the same to the storages. Then the united wind farm and united storage participates separately as price takers. Similarly, the profit of coordination is also calculated.

Aggregation with PM strategy. After aggregation, the united WF and ESS participate separately as price makers, and the profit of WF-ESS coordination is also studied.

The results of these three cases are listed in Tab.III, Tab.IV and Tab.V. The results are analyzed and compared as follows.

The profit of distributed WF and ESS is shown as Tab. III, where Row 2 and Row 3 are the profit of distributed WF, Row 4 and Row 5 are profit of distributed ESS, and Row 6 and Row 7 are the profit of distributed WF-ESS. Row 2, 4 and 6 are results of objective function while Row 3,5 and 7 are results of simulation.
TABLE III

PROFIT OF DISTRIBUTED WF AND ESS $(\mathrm{k} €)$

\begin{tabular}{cccc}
\hline Item & Day-ahead Profit & Balancing Profit & Overall Profit \\
\hline WF only Obj. & 28.17 & -1.15 & 27.02 \\
WF only & 26.98 & -1.65 & 25.33 \\
Simulation & -0.90 & 2.30 & 1.40 \\
ESS only Obj. & -2.17 & 1.47 & -0.70 \\
ESS only & 27.28 & 1.15 & 28.43 \\
Simulation & 24.81 & 0.76 & 25.57 \\
WF-ESS Obj. & & & \\
WF-ESS & & & \\
Simulation & &
\end{tabular}

TABLE IV

PRofit OF AGgREgated WF AND ESS WITH THE PT STRATEgy $(k €)$

\begin{tabular}{cccc}
\hline Item & Day-ahead Profit & Balancing Profit & Overall Profit \\
\hline WF only Obj. & 281.74 & -11.53 & 270.21 \\
WF only & 269.83 & -16.51 & 253.32 \\
Simulation & -8.98 & 23.06 & 14.08 \\
ESS only Obj. & -21.69 & 8.92 & -12.77 \\
ESS only & 272.76 & 11.52 & 284.29 \\
Simulation & 248.14 & -18.83 & 229.31 \\
WF-ESS Obj. & & & \\
WF-ESS & & & \\
Simulation & &
\end{tabular}

It can be seen from the table that profit of WF mainly comes from the day-ahead market while that of ESS mainly comes from balancing market. The simulation results of ESS shows its overall profit is negative, which means small scaled ESS cannot benefit from energy markets by proposed strategies. The simulated profit of WF-ESS is $25.57 \mathrm{k} €$, higher than the total profit of WF and ESS without coordination (25.33$0.7=24.63 \mathrm{k} €$ )

Tab IV lists the results of aggregated WF and ESS with the PT strategy. Results for the united WF are accurately 10 times of those for distributed WFs. But the simulated balancing profit for united ESS is less than 10 times of the distributed ESS, which is because that the key parameters of linear decision rules change with the capacity of ESS. The united WF-ESS gets $229.31 \mathrm{k} €$, less than the sum of distributed WF-ESS $(253.32-12.77=240.55 \mathrm{k} €)$.

TABLE V

Profit of AGgRegated WF AND ESS With the PM StRategy $(\mathrm{k} €)$

\begin{tabular}{cccc}
\hline Item & Day-ahead Profit & Balancing Profit & Overall Profit \\
\hline WF only Obj. & 125.68 & 142.63 & 268.31 \\
WF only & 121.57 & 139.17 & 260.74 \\
Simulation & 19.82 & -8.36 & 11.46 \\
ESS only Obj. & 17.28 & -24.60 & -7.32 \\
$\quad$ ESS only & 91.55 & 186.69 & 278.24 \\
Simulation & 91.55 & 190.43 & 281.98 \\
WF-ESS Obj. & & & \\
WF-ESS & & & \\
Simulation & & &
\end{tabular}

Tab.V shows the results of united WF and ESS with the PM strategy. Comparing Row 2 with Row 3, and Row 4 with Row 5, one can find that although the united WF and ESS considers the influence of offering quantity on cleared prices, as they do not know other participants' offering, the simulated day-ahead profit is still lower than that from the model, but the decrease is much smaller than the results of PT strategy. The 
profit of coordinated WF-ESS is $281.98 \mathrm{k} €$ (Row 7), $11.3 \%$ higher than the profit of uncoordinated WF-ESS $(253.42 \mathrm{k} €$, adding the results of Row 3 and Row 5). With Tab.V and Tab.IV compared, it can be seen that PM strategy can help WF increase profit by $2.9 \%$, while help ESS reduce the loss by $42.7 \%$. For the distributed resources, their profit can be increased by $14.49 \%$ if aggregated by VPP and participate in electricity markets with the PM strategy.

By comparing Row 4 and Row 5 of Tab. III, IV and V, we can find that the objective function values of ESS profit ("ESS only Obj.") are all positive, and the simulation results ("ESS only Simulation") are negative, no matter it takes PM or PT strategy. This is due to the decrease of both day-ahead and balancing profit. On one hand, wind farms' offers will decrease the cleared prices, which makes the day-ahead profit of ESS lower. On the other hand, as the parameters of linear decision rules are optimized based on a given set of scenarios, the balancing profit for out-of-sample scenarios will decrease. These two reasons make the simulation profit negative.

TABLE VI

Daily Profit of WF-ESS BRought B y the PT and PM STRategies $(\mathrm{k} €)$

\begin{tabular}{ccccccc}
\hline Month & Jan. & Feb. & Mar. & Apr. & May & June \\
PT Strategy & 258.51 & 294.20 & 266.73 & 257.12 & 221.80 & 192.56 \\
PM Strategy & 301.31 & 320.65 & 293.48 & 280.65 & 249.76 & 222.85 \\
\hline Month & July & Aug. & Sep. & Oct. & Nov & Dec. \\
PT Strategy & 137.37 & 220.63 & 213.88 & 264.35 & 253.42 & 190.40 \\
PM Strategy & 186.57 & 285.13 & 241.19 & 289.23 & 281.98 & 214.09 \\
\hline
\end{tabular}

By calculating the profit of one typical day in every month in 2015, Tab.VI lists the profit of WF-ESS brought by PM and PT strategies throughout the year. During the whole year, PM strategy can bring $14 \%$ more profit than PT strategy for WF-ESS, which validates the effectiveness of our proposed strategy.

\section{CONClusion}

This paper proposes an optimal offering and operating strategy of WF-ESS as a price maker in day-ahead markets and adopts linear decision rules in balancing markets. A mixed integer linear programming is modeled accordingly and this model can integrate control strategy of balancing stage into day-ahead optimization, which helps guarantee the optimality of overall revenue.

Case studies based on realistic data of wind farms and electricity markets are carried out. The results show that over $60 \%$ profit of WF-ESS comes from the balancing market when it participates in day-ahead markets as a price maker. The price-maker strategy can help WFs, ESS and WF-ESS achieve more profit than the price-taker strategy. Furthermore, if there are many distributed wind and storage resources, aggregating them to a VPP and adopting the price-maker strategy can increase their profit by about $10 \%$.

\section{REFERENCES}

[1] H. Ding, P. Pinson, Z. Hu, and Y. Song, "Optimal offering and operating strategies for wind-storage systems with linear decision rules," IEEE Trans. Power Syst., vol. 31, no. 6, pp. 4755-4764, Nov 2016. [Online]. Available: http://ieeexplore.iee.org/stamp/stamp.jsp?tp=\&arnumber=7398187
[2] J. Garcia-Gonzalez, R. M. R. de la Muela, L. M. Santos, and A. M. Gonzalez, "Stochastic joint optimization of wind generation and pumped-storage units in an electricity market," IEEE Trans. Power Syst., vol. 23, no. 2, pp. 460-468, 2008. [Online]. Available: http://ieeexplore.ieee.org/stamp/stamp.jsp?tp=\&arnumber $=4494596$

[3] L. S. Vargas, G. Bustos-Turu, and F. Larrain, "Wind power curtailment and energy storage in transmission congestion management considering power plants ramp rates," IEEE Trans. Power Syst., vol. 30, no. 5, pp. 2498-2506, 2015. [Online]. Available: http://ieeexplore.ieee.org/stamp/stamp.jsp?tp=\&arnumber $=6936947$

[4] H. Ding, P. Pinson, Z. Hu, and Y. Song, "Integrated bidding and operating strategies for wind-storage systems," IEEE Trans. Sustain. Energy, vol. 7, no. 1, pp. 163-172, 2016. [Online]. Available: http://ieeexplore.ieee.org/lpdocs/epic03/wrapper.htm?arnumber=7296676

[5] E. D. Castronuovo and J. A. P. Lopes, "Optimal operation and hydro storage sizing of a wind-hydro power plant," Inter. Jour. of Elect. Power \& Energy Syst., vol. 26, no. 10, pp. 771-778, 2004. [Online]. Available: http://www.sciencedirect.com/science/article/pii/S0142061504000870

[6] S. R. Dabbagh and M. K. Sheikh-El-Eslami, "Risk assessment of virtual power plants offering in energy and reserve markets," IEEE Transactions on Power Systems, vol. 31, no. 5, pp. 3572-3582, Sept 2016.

[7] B. Zhou, X. Liu, Y. Cao, C. Li, C. Y. Chung, and K. W. Chan, "Optimal scheduling of virtual power plant with battery degradation cost," IET Generation, Transmission Distribution, vol. 10, no. 3, pp. 712-725, 2016.

[8] Q. Zhao, Y. Shen, and M. Li, "Control and bidding strategy for virtual power plants with renewable generation and inelastic demand in electricity markets," IEEE Transactions on Sustainable Energy, vol. 7, no. 2, pp. 562-575, April 2016.

[9] M. Rahimiyan and L. Baringo, "Strategic bidding for a virtual power plant in the day-ahead and real-time markets: A price-taker robust optimization approach," IEEE Transactions on Power Systems, vol. 31, no. 4, pp. 2676-2687, July 2016.

[10] L. Baringo and A. J. Conejo, "Strategic offering for a wind power producer," IEEE Trans. Power Syst., vol. 28, no. 4, pp. 4645-4654, 2013. [Online]. Available: http://ieeexplore.ieee.org/xpl/articleDetails.jsp?tp=\&arnumber $=6571288$

[11] A. Conejo, J. Contreras, J. Arroyo, and S. de la Torre, "Optimal response of an oligopolistic generating company to a competitive pool-based electric power market," IEEE Trans. Power Syst., vol. 17, no. 2, pp. 424-430, may 2002. [Online]. Available: http://ieeexplore.ieee.org/lpdocs/epic03/wrapper.htm?arnumber=1007913

[12] S. De La Torre, J. M. Arroyo, A. J. Conejo, and J. Contreras, "Price maker self-scheduling in a pool-based electricity market: A mixed-integer LP approach," IEEE Trans. Power Syst., vol. 17, no. 4, pp. 1037-1042, 2002. [Online]. Available: http://ieeexplore.iee.org/stamp/stamp.jsp?tp=\&arnumber $=1137591$

[13] C. Ruiz and A. J. Conejo, "Pool strategy of a producer with endogenous formation of locational marginal prices," IEEE Trans. Power Syst., vol. 24, no. 4, pp. 1855-1866, 2009. [Online]. Available: http://ieeexplore.ieee.org/stamp/stamp.jsp?tp=\&arnumber $=5272241$

[14] M. Zugno, J. M. Morales, P. Pinson, and H. Madsen, "Pool strategy of a price-maker wind power producer," IEEE Trans. Power Syst., vol. 28, no. 3, pp. 3440-3450, 2013. [Online]. Available: http://ieeexplore.ieee.org/xpl/articleDetails.jsp?tp=\&arnumber $=6494365$

[15] C. G. Baslis and A. G. Bakirtzis, "Mid-term stochastic scheduling of a price-maker hydro producer with pumped storage," IEEE Trans. Power Syst., vol. 26, no. 4, pp. 1856-1865, 2011. [Online]. Available: http://ieeexplore.ieee.org/stamp/stamp.jsp?tp=\&arnumber $=5734883$

[16] S. J. Kazempour, A. J. Conejo, and C. Ruiz, "Strategic bidding for a large consumer," IEEE Trans. Power Syst., vol. 30, no. 2, pp. 848-856, 2015. [Online]. Available: http://ieeexplore.ieee.org/stamp/stamp.jsp?tp=\&arnumber $=6850076$

[17] J. M. Morales Gonzlez, A. J. Conejo, H. Madsen, P. Pinson, and M. Zugno, Integrating Renewables in Electricity Markets: Operational Problems, ser. International Series in Operations Research and Management Science. Springer, 2014.

[18] C. Ruiz, A. J. Conejo, and D. J. Bertsimas, "Revealing rival marginal offer prices via inverse optimization," Power Systems, IEEE Transactions on, vol. 28, no. 3, pp. 3056-3064, 2013, power Systems, IEEE Transactions on.

[19] P. Pinson, "Wind energy: Forecasting challenges for its operational management," Statistical Science, vol. 4, no. 28, pp. 564-585, 2013.

[20] W. Bukhsh, C. Zhang, and P. Pinson, "An integrated multiperiod opf model with demand response and renewable generation uncertainty," IEEE Trans. Smart Grid, vol. PP, no. 99, pp. 1-9, 2015. 
[21] W. Bukhsh, "Wind scenarios - data for stochastic multi-period optimal power flow problem," http://sites.google.com/site/datasmopf/windscenarios.

[22] Energinet, "Download of market data [online]," http://energinet.dk/EN/El/Engrosmarked/Udtraek-afmarkedsdata/Sider/default.aspx.

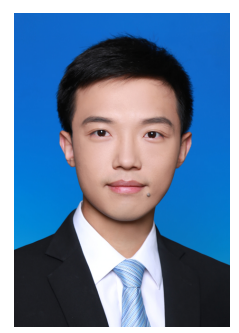

Huajie Ding (M'16) received the Bachelor's degree and Ph.D. degree from the Department of Electrical Engineering at Tsinghua University (THU), Beijing, China, in 2011 and 2016, respectively.

He currently works in State Grid Jibei Electric Power Company, Beijing, China. His research interests include renewable energy integration, energy storage systems, power system modeling and optimization.

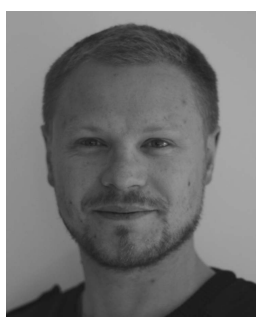

Pierre Pinson (M'11-SM'13) received the M.Sc. degree in applied mathematics from the National Institute for Applied Sciences (INSA Toulouse, France) and the Ph.D. degree in energetics from Ecole des Mines de Paris (France).

$\mathrm{He}$ is a Professor at the Technical University of Denmark, Centre for Electric Power and Energy, Department of Electrical Engineering, also heading a group focusing on Energy Analytics \& Markets. His research interests include among others forecasting, uncertainty estimation, optimization under uncertainty, decision sciences, and renewable energies. Prof. Pinson acts as an Editor for the International Journal of Forecasting, and for Wind Energy.

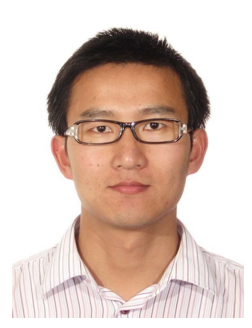

Zechun Hu (M'09-SM'17) received the B.S. degree and $\mathrm{Ph} . \mathrm{D}$. degree from Xian Jiao Tong University, Shaanxi, China, in 2000 and 2006, respectively.

He worked in Shanghai Jiao Tong University after graduation and also worked in University of Bath as a research officer from 2009 to 2010 . He joined the Department of Electrical Engineering at Tsinghua University in 2010 where he is now an associate professor. His major research interests include optimal planning, operation of power systems and electric vehicles.

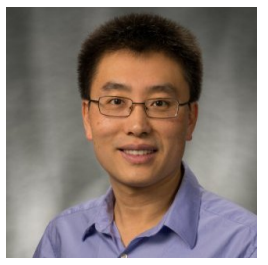

Jianhui Wang (M'07-SM'12) received the Ph.D. degree in electrical engineering from Illinois Institute of Technology, Chicago, Illinois, USA, in 2007. Presently, he is an Associate Professor with the Department of Electrical Engineering at Southern Methodist University, Dallas, Texas, USA.

Dr. Wang is the secretary of the IEEE Power \& Energy Society (PES) Power System Operations, Planning \& Economics Committee. He is an associate editor of Journal of Energy Engineering and an editorial board member of Applied Energy. He has held visiting positions in Europe, Australia and Hong Kong including a VELUX Visiting Professorship at the Technical University of Denmark (DTU). Dr. Wang is the Editor-in-Chief of the IEEE Transactions on Smart Grid and an IEEE PES Distinguished Lecturer. He is also the recipient of the IEEE PES Power System Operation Committee Prize Paper Award in 2015.

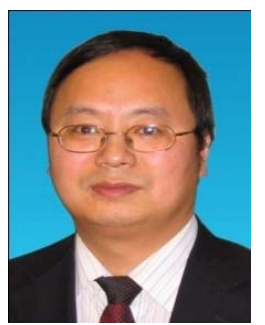

Yonghua Song (F'08) received his BEng and $\mathrm{PhD}$ from Chengdu University of Science and Technology, and China Electric Power Research Institute in 1984 and 1989 respectively. He was a Postdoctoral Fellow at Tsinghua University from June 1989 to March 1991.

In 2004, he was elected Fellow of the Royal Academy of Engineering (UK). In 2008, he was elected Fellow of the Institute of Electrical and Electronics Engineers (USA). He returned to Tsinghua University in February 2009 as a Professor at the Department of Electrical Engineering. His research areas include Smart Grid, electricity economics, and operation and control of power systems. 